\title{
During Introduction of Mammography Screening Analysis of Three Tumor Size Intervals in Screened and Post-Screened Periods Clarified the Short and Long Term Efficacy of Screening
}

Roland B Sennerstam*

Department of Oncology and Pathology Karolinska Hospital and Karolinska Institutet Stockholm, Sweden

\begin{abstract}
The present study focused on mammography screening during two periods representing 2 years (1991) to 8 years (1997/98) after the introduction of screening in 1989 in the Stockholm Gotland County, Sweden for women aged 50-69 years. Subjects were sorted by age, as younger ( $<50$ years) unscreened women, screening women aged 50-69 years and analyzed in two decades between 50-59 years and 60-69 years respectively, and unscreened women of $\geq 70$ years. Tumor size was compared with an unscreened cohort from 1987, 2 years before screening was introduced. Resolution was increased by focusing on three tumor-size intervals instead of mean tumor size, which showed a tending reduction in tumors $\geq 20 \mathrm{~mm}$ for women diagnosed with breast cancer aged 50-59 years in the 1991 sample but a significant decline for women aged 60-69 years having attended two screening tests. After 8 years of biennial screening (1997/98) patients with tumor size $\leq 10 \mathrm{~mm}$ at diagnosis had significantly increased, and tumors $\geq 20 \mathrm{~mm}$ declined significantly-most clearly among 60-69-year-old women after attending up to five screening tests. A transient increase in tumor sized $10 \mathrm{~mm}$ to $20 \mathrm{~mm}$ was seen due to the stepwise-altered distribution in size. Women in the two unscreened age groups $<50$ years and $\geq 70$ years, were compared with screened women aged 60-69 (1997/98) according to tumor size, genomic instability, proliferation index, lymph node metastases, cyclin-A and ki67, alteration in breast tumor stage I and stage IIB and survival rate. In all parameters except ki67, only in relation to unscreened women $<50$ years old showed a significant reduction. A post-screening effect was also found for women aged 70-79 years with tumors size $>20 \mathrm{~mm}$ still being reduced compared with controls and the tumors $\leq 10 \mathrm{~mm}$ decreasing significantly to the control level.
\end{abstract}

Keywords: Mammography screening; Post-screening effect; Tumor size; Molecular profiles; Death rate; Over diagnosis

Abbreviations: PI :Proliferation Index; $\mathrm{G}_{1} \mathrm{CV}$ : $\mathrm{G}_{1}$ coefficient of variation; SSI : Stemline-Scatter-Index; SPF : S-phase; ALNM : Axillary Lymph Node Metastasis; CBE : Clinical Breast Cancer Examination; BSE : Breast Self Examination; MM : Mammography Screening; DMC: Dedicated Mammography Screening Center; GP : General Radiology; M : Mobile Unit

\section{Introduction}

Mammography screening has been the subject of debate ever since it was first introduced. A follow-up data on breast cancer incidence in five countries and three continents after introduction of general screening revealed an increase of breast cancer incidence of $52 \%[1,2]$. The similar phenomenon has been reported from Sweden $[3,4]$. A reduction in mortality by $31 \%$ and $25 \%$ decline in the rate of tumor stage II or more advanced breast cancer were found in two Swedish counties between the start of screening and a 7years of follow-up of about 165000-screened women [5]. Efficiency of screening by age showed a smaller positive effect in women aged 40-49 compared with older women [6] Furthermore, data from 1972-2009 show breast cancer mortality rates decreased from 68.4 to 42.8 per 100,000 women and continue to decline in 14 of the 21 counties in Sweden [7]. In the beginning of the 1970s, the first counties in Sweden started to invite women to mammography screenings. During the following 23 years, the rest of the counties planned and introduced general screening. A recent report describes 18 years follow-up of mortality in breast cancer during this introduction period. Counties that first introduced screening programs during 1974-1978 had no improvement in survival and during 1986-1987, mortality increased with $12 \%$. After 1987, the results began to improve. In the follow-up of the cohorts screened from $1987-1988$, mortality decreased by $5 \%(\mathrm{p}<0.01)$; among breast cancer patients diagnosed from $1989-1990$, mortality was reduced by $8 \%(\mathrm{p}<0.01)$ [7]. Survival advantage for women with screen-detected tumors has continued to be reported in studies the last decade [8-10]. The early data during the introduction period of screening may reflect the initial effort to get the technology in place, assistants trained and logistics working.

A follow up of a well-screened population in Rhode Island, USA over four time periods diagnosed between 1987 and 2008 revealed statistically significant improvements in reduction of mean cancer size, more favorable pathologic grade, increased breast-conserving surgery and decline in mortality [11]. Other studies suggest that some smaller tumors might not have come to a clinical stage during the woman's life time, which evokes a debate about overdiagnosis $[1,12,13]$. An early study reported increased Stage II-IV breast tumors to be found in a study group during the first 3-4 years after introduction of screening but not in the control group, indicating a spontaneous regression of some breast tumors [14]. A proposed natural history of breast cancer includes the idea that some tumors regress spontaneously [15]; such

*Corresponding author: Roland B Sennerstam, Department of Oncology and Pathology Karolinska Hospital and Karolinska Institutet Stockholm, Sweden, Tel: +46 (0) 707810201; Fax: 00468331 696; E-mail: roland.sennerstam@ki.se

Received June 12, 2013; Accepted June 28, 2013; Published July 01, 2013

Citation: Sennerstam RB (2013) During Introduction of Mammography Screening Analysis of Three Tumor Size Intervals in Screened and Post-Screened Periods Clarified the Short and Long Term Efficacy of Screening. J Cancer Sci Ther S7: 006. doi:10.4172/1948-5956.S7-006

Copyright: ( $) 2013$ Sennerstam RB. This is an open-access article distributed under the terms of the Creative Commons Attribution License, which permits unrestricted use, distribution, and reproduction in any medium, provided the original author and source are credited. 
results may be considered false-positive cases [16]. Analyzing 10year survival data in almost 20000 women aged 50-65 years in West Midlands region UK showed that a combination of lead time with tumor mean size and lymph node status in 10 categories could explain $97 \%$ of survival advantage in favor of screened-detected versus symptomatically diagnosed breast cancers. Only a small proportion remained to be explained by length bias and overdiagnosis [17].

Lately, age group in the Stockholm Gotland area has extended mammography screening; by 2005 , it included women as young as 40 years old, and in 2012, older women up to 74 years of age were included. Guideline programs for mammography screening, type of organization and methods to detect breast cancer from four countries on two continents in service in 1995 (i.e., contemporary to our study period) are shown in Table 1 [18]. The comparison shows that Sweden was well organized in dedicated mammography screening centers organized in line with a common centralized system. The digital mammography technique was introduced in the Stockholm Gotland area in the years 2006 to 2008.

Because some findings from the introduction period of mammography are not clearly understood, this report follows alterations in screening three tumor- size intervals and molecular profiles [19] to compare the unscreened and screened women diagnosed with breast cancer. The post-screening period is also included, which may provide insights to evaluate the overall benefits of mammography screening over time and changes in progressive improvements.

\section{Materials and Methods}

To reflect the first decade after introduction of mammography screening in 1989 in the Stockholm- Gotland area, cytochemical parameters and survival data was analyzed 2 and 8 years later. Two consecutive retrospective samples of breast cancer patients from 1991 $(n=519)$ and 1997/98 $(n=345)$ were examined at the department of Oncology and Pathology, Karolinska University Hospital, Sweden. All patients were treated by either quadrantectomy or wide excision and no wire-localization of small tumors was used. Diagnoses were confirmed after radiography by fine needle biopsy before surgery. The few DCIS found were excluded. The two tumor samples were analyzed for tumor size, proliferation index (PI), including S-phase+G2-phase, and genomic instability reflected as Stemline-Scatter-Index (SSI) (see below). Axillary lymph node metastases were investigated after excision of the axillary tissue. The use of sentinel nodes was not in practice at this time. Tumor stages I and IIB were ascertained. Cyclin-A and k67 testing were in use only in the 1997/98 sample. DNA-image analysis procedures were done at about in time of diagnosis. One tumor sample from the unscreened period in 1987 was used to compare tumor size with the two screened groups. A number of 445 consecutive breast tumors from 1987, of which 400 had information on tumor size, were selected as controls. We used three tumor size intervals $(\leq 10 \mathrm{~mm}$ $10-20$ and $>20 \mathrm{~mm}$ ) to reflect alterations in screening effect. Data on deaths due to breast cancer and other causes were retrieved from the Swedish Cause of Death Register. Permission to analyze the samples from clinical patient data was obtained by the Ethical Committee Nord, Karolinska Institutet (2010/34-31/1).

\section{Feulgen staining}

To examine genomic instability and proliferation activity the DNA parameters in Feulgen-stained tumor nuclei were used. Nuclear DNA content was measured by image cytometry in Feulgen-stained cell nuclei mainly from imprints and some on $8 \mu \mathrm{m}$ histological sections from the primary breast adenocarcinoma. A pathologist identified single tumor cells; 200 cells per slide were analyzed. Materials were rehydrated in an ethanol series and hydrolysis in one batch of $5 \mathrm{M} \mathrm{HCL}$ at $22^{\circ} \mathrm{C}$ for $60 \mathrm{~min}$. Specimens were then rinsed in distilled water and stained with Shiff reagent at $22^{\circ} \mathrm{C}$ for $90 \mathrm{~min}$. Normal lymphocyte cells were used as an internal diploid DNA standard (2C). The cells were washed three times in sulfurous acid $\left(10 \mathrm{ml} \mathrm{Na}_{2} \mathrm{~S}_{2} \mathrm{O}_{5}\right)$. Stained cells were then measured in a computer assisted image-analysis system based on an axioscope (Zeiss, Baden-Württenberg, Germany), equipped with an immersion plan-objective (40/0,95; Nikon, Tokyo, Japan) and a CCD camera (Nikon).

\section{Stemline-scatter-index (SSI)}

In several reports, we used a large-scale estimation for genomic instability and proliferative activity [20], based on parameters from Feulgen-DNA stained nuclei. The parameters combined are the coefficient of variation for the $G_{1}$ peak $\left(G_{1} C V\right)$ of tumor DNA stemlines (DI) and DNA content values in the S-phase fraction (SPF) for each patient, plus the percentage of cells with DNA content above the $G_{2}$ DNA level (exceeding $G_{2}$ rate, $E_{x} G_{2}$ ). Thus, the StemlineScatter-Index (SSI) includes $\mathrm{G}_{1} \mathrm{CV}+\mathrm{SPF}+\mathrm{ExcG}_{2}$ all expressed in percentages. In previous reports, we used the cut-point value of SSI $=8.8 \%$ to differentiate between tumors representing significantly scattered DNA histograms (SSI $>8.8 \%$ ) and those with insignificantly scattered ones (SSI $\leq 8.8 \%$ ). Breast carcinoma with a SSI $\leq 8.8 \%$ were classified as genomic-stable and those with a SSI $>8.8 \%$ as genomicunstable. However, as increased genomic instability and proliferative activity represent a continuum a cut-point is a somewhat artificial for classifying tumors.

\begin{tabular}{|c|c|c|c|c|c|c|c|}
\hline $\begin{array}{l}\text { Countries } \\
\text { compared }\end{array}$ & $\begin{array}{l}\text { First national } \\
\text { pro-gram }\end{array}$ & $\begin{array}{l}\text { Age group } \\
\text { screened (years) }\end{array}$ & $\begin{array}{l}\text { Time interval } \\
\text { between MM } \\
\text { (years) }\end{array}$ & $\begin{array}{l}\text { Detec-tion } \\
\text { method }\end{array}$ & $\begin{array}{l}\text { Organized around } \\
\text { the country }\end{array}$ & $\begin{array}{l}\text { Type of MM } \\
\text { scree-ning centre }\end{array}$ & $\begin{array}{l}\% \text { target popu- } \\
\text { lation within } \\
\text { screening program }\end{array}$ \\
\hline Sweden & 1986 & $50-69$ & 2 & MM & Partially centra-lized & DMC & $100 \%$ \\
\hline Canada & 1988 & $50-69$ & 2 & $\begin{array}{l}\text { MM } \\
\text { CBE } \\
\text { BSE }\end{array}$ & De-centra-lized & DMC & $<25 \%$ \\
\hline United States & 1991 & $\begin{array}{l}40-50 \\
50+\end{array}$ & $\begin{array}{l}1 \\
1-2\end{array}$ & $\begin{array}{l}\text { MM } \\
\text { CBE } \\
\text { BSE }\end{array}$ & $\begin{array}{l}\text { Partially } \\
\text { centra-lized }\end{array}$ & $\begin{array}{l}\text { DMC } \\
\text { GR } \\
\text { M }\end{array}$ & $20-50 \%$ \\
\hline United Kingdom & 1988 & $50-64$ & 3 & MM & Partially centra-lized & $\begin{array}{l}\text { DMC } \\
\text { M }\end{array}$ & $100 \%$ \\
\hline
\end{tabular}

First national mammography screening programs for four countries on two continents are compared for the period of our study, including initial years, target age groups, time intervals between screenings, and type of detection (MM, mammography screening; CBE, clinical breast examination; BSE, breast self-examination). Screening center administrations that were partly centralized were administrated regionally; guided by national policies but with regional funding and administration. Types of facilities were DMC, dedicated mammography screening center, GR, general radiology department; M, mobile unit (from ref. 12).

Table 1: Guidelines in four countries 1995 
Citation: Sennerstam RB (2013) During Introduction of Mammography Screening Analysis of Three Tumor Size Intervals in Screened and PostScreened Periods Clarified the Short and Long Term Efficacy of Screening. J Cancer Sci Ther S7: 006. doi:10.4172/1948-5956.S7-006

Page 3 of 7

\section{Immunohistochemistry}

Tumor samples were fixed in $4 \%$ phosphate buffered formaldehyde directly after operation and paraffin embedded. From each specimen, contiguous $4 \mu \mathrm{m}$ sections were prepared and used on HE staining and immunohistochemistry. Sections were deparaffinized with xylene, rehydrated through a graded alcohol series and microwaved at $500 \mathrm{~W}$ for 2 x $5 \mathrm{~min}$ in $10 \mathrm{mM}$ citrate buffer ( $\mathrm{pH}$ 6.0). After rinsing in Trisbuffered saline (TBS, pH 7.6), sections were treated with $3 \%$ hydrogen peroxide in methanol to exhaust endogenous peroxidase activity followed by normal horse serum (1:20 dilution) in $0.1 \mathrm{M} \mathrm{PBS} \mathrm{(pH} \mathrm{6.0),}$ and the incubated overnight with the monoclonal primary antibodies diluted in $1 \%$ (wt/vol) bovine serum albumin (BSA) and visualized by standard avidin-biotin-peroxidase complex technique (Vector Laboratories, Burlingame, CA). Counterstaining was performed with Mayer's hematoxylin. The antibodies used were MIB-1 (antibody against the nuclear proliferation associated antigen Ki67, Immunotech S.A., Marseille, France) dilusion 1:150; NCL-cyclin A ( Cyclin A monoclonal antibody, Novocastra Laboratories Ltd, Newcastle upon Tyne, UK; dilution 1:100). Only distinct nuclear staining was accepted as a positive reaction. All cells with simultaneous nuclear and cytoplasmic cyclin A staining were regarded as positive for cyclin $\mathrm{A}^{+}$.

\section{Lymph Node Metastasis}

In this study, the fraction (0-1) of axillary lymph node metastases (ALNM) was used in place of counted positive glands (i.e., where cancer cells were found). In 1991 cohort yielded 519 patients from whom 303 surgical specimens were taken (58\%) Lymph node metastases $\left(\mathrm{ALNM}^{+}\right)$were observed in 124 patients $(24 \%)$. The mean number of lymph nodes counted per patient in this group was $8.9 \pm 4.2$. Among lymph node negative axillary surgical extractions $\left(\mathrm{ALNM}^{-}\right)$, the mean number of counted glands was $8.4 \pm 3.6(n=179)$. From the $1997 / 98$ cohort, 218 of 354 (63\%) lymph node axillary extractions were done and $76(22 \%)$ were $\mathrm{ALNM}^{+}$. The mean number of node counted in the $\mathrm{ALNM}^{+}$group was $9.4 \pm 3$ 3. In the negative lymph node group $\left(\mathrm{ALNM}^{-}\right)$the mean number of nodes counted was10.5 $\pm 43.6(\mathrm{n}=139)$. No statistical difference was found between mean counted nodes in the $\mathrm{ALNM}^{+}$and $\mathrm{ALNM}^{-}$groups in either patient sample, which justified use of ALNM fractions. In estimating tumor stages I and IIB, only patients with ALNM+ findings were included (TNM staging system AJCC-6). As only lymph node classification, N1 (1-3 positive nodes found) is represented in stages IIB, an upper limit for the fraction and I was put at $\mathrm{ALNM}^{+}<0.4$.

\section{Statistical Analysis}

Statistical calculations were performed using the STATISTICA software package (StatSoft, Inc., Tulsa, OK, USA). Statistical significance for categorical variables was calculated using the chi square test and an independent $t$-test was used for continuous ones. Linear regression was performed for the correlation test. Statistical significance was assumed if $p<0.05$.

\section{Results}

By analyzing two samples with 8 year's difference within the first decade after introduction of mammography screening, the effect of screening can be followed step by step. The individual patients diagnosed with breast cancer in the sample from 1997/98 were involved in the screening for 8 years at most; and the patients from 1991 for 2 years, since start of the biennial screening in 1989.

The four patient age groups showed a significant reduction in mean tumor size only in the screened women diagnosed with breast cancer aged 60-69 years from both 1991 and 1997/98, compared with the unscreened age groups from 1987 (Table 2).

To reach a stronger resolution, relative distributions within three tumor- size intervals (see Materials and Methods section) were analyzed in comparison between the two screened breast cancer patients from 1991 and 1997/98 versus the control sample from 1987 (Figures $1 \mathrm{a}$ and $1 \mathrm{~b}$ ). The screened breast cancer women aged 50-59 years in the 1991 sample revealed no significant deviation from the controls. They tended to decline for tumors $>20 \mathrm{~mm}$, but only those in the age group 60-69 showed a statistical significant reduction (Figure 1b). The 1997/88 sample showed significantly fewer tumors $>20 \mathrm{~mm}$ and significantly higher percentage of tumors $\leq 10 \mathrm{~mm}$ compared with controls in both screened ages (Figures 1a and 1b); this effect was stronger in the screened patients aged 60-69 years (Figure 1b).

In the 1991 sample, for screened patients aged 60 to 69 years, the percentage of tumors $10-20 \mathrm{~mm}$ increased significantly both related to the controls and to the $1997 / 98$ sample (Figure 1b). This was a transient relative increase for the 1991 sample as tumors $\leq 10 \mathrm{~mm}$ have not yet been diagnosed to an increased degree, whereas the percentage of tumors $>20 \mathrm{~mm}$ had decreased significantly.

\section{Tumor stage IIA T1c}

The transient increase in tumors $10-20 \mathrm{~mm}$ in the 1991 sample is included in tumor Stage IIA T1c. In the 1991 sample, 164 women were diagnosed as Stage IIA T1 a-c breast cancers, 87 women in Stage IIA T1c (53\%) and 54 women were diagnosed as Stage IIB T2-3, including tumor size $>20 \mathrm{~mm}$ (see Discussion).

\section{Age and tumor size reflected in 2-dimentional plots}

To show changes in tumor size, two-dimentional plots are shown in Figure 2.The control group from 1987 was compared with the 1997/98 sample in the tumor age interval $\leq 65$ years. In the $1997 / 98$ sample, the plot reflects the decline in tumor size for screened patients up to 65 years of age. The negative slope of the curve for the 1997/98 sample is statistically significant (Figure 2b). A similar comparison to the 1991

\begin{tabular}{|c|c|c|c|c|c|}
\hline Age in Years (n) & 1991 Size mm (n) & $P$ & 1987 Size mm (n) & $P$ & 1997/98 Size mm (n) \\
\hline$<50(96)$ & $19.4 \pm 12.6$ (80) & ns & $22.0 \pm 13.8$ (98) & ns & $22.8 \pm 13.5(67)$ \\
\hline $50-59(102)$ & $19.3 \pm 13.6(96)$ & ns & $21.6 \pm 11,9$ (79) & ns & $18.5 \pm 12,9(119)$ \\
\hline $60-69(142)$ & $17.7 \pm 13.8(130)$ & $<0.001$ & $23.5 \pm 13,3(120)$ & $p<0.0005$ & $15.4 \pm 12.4(70)$ \\
\hline$\geq 70$ (169) & $21.8 \pm 11.5(108)$ & ns & $22.5 \pm 13.3(103)$ & ns & $20.4 \pm 14.2(89)$ \\
\hline
\end{tabular}

Tumors from a control sample from 1987 and samples from 1991 and 1997/98 are sorted to four age intervals. The two screened age groups are flanked by those younger than 50 years of age and those older than 70 years, neither of which were invited to the screening. The results shows a significant reduction of mean tumor size related to screening only for women aged 60 to 69 years for both 1991 and 1997/98-samples.

Table 2: Tumor size in four age intervals in comparison of two screened cohorts against controls. 
Citation: Sennerstam RB (2013) During Introduction of Mammography Screening Analysis of Three Tumor Size Intervals in Screened and PostScreened Periods Clarified the Short and Long Term Efficacy of Screening. J Cancer Sci Ther S7: 006. doi:10.4172/1948-5956.S7-006

sample was not significant $(P=0.32$; not shown). In the control group no correlation was seen between tumor size and age (Figure 2a).

\section{Tumor size PI SSI ALMN+ Cyclin-A. Ki_67 Stage I Stage IIB}

The best screening effect was found in the 60-69 year age group in the 1997/98 sample, when the women had attended to screening tests about five times, and for whom samples were analyzed according to proliferation index (PI), SSI, lymph node metastasis, cyclin-A, ki67 and tumor stage I and IIB in relation to the two unscreened patients aged $<50$ years and $\geq 70$ years. For all parameters except ki67, the screened patients aged 60-69 years differed significantly from the younger unscreened group aged $<50$ years. However, the percentage of ki67 ${ }^{+}$ cells differed between the two unscreened age groups $(<50$ and $\geq 70$ years; $P<0.001$ ) with significantly higher values for woman aged $<50$ years reflecting highly proliferative tumors among young women in contrast to more slower growing tumors in older women. For stage I and stage IIB tumors a significant higher percentage of stage I tumors was found in the screened group aged 60-69 years as compared with the unscreened group $<50$ years. Furthermore the tumor Stage IIB group was significantly reduced in the screened sample versus the unscreened group $<50$ years. No significant differences emerged in any parameter over the older age group (Table 3). A similar investigation done for patients from 1991 showed the younger control group differed significantly only regarding lymph node metastasis $(P<0.05)$ and reduction in tumor Stage IIB in the 1991 sample $(P<0.05$; data not shown).

\section{Reduction in breast cancer death rate}

Screened woman diagnosed with breast cancer aged 60-69 years was studied with respect to death and survival rates versus the two unscreened breast cancer age groups over ten years of followup (Table 4). Screened groups from both 1991 and from 1997/98 show significantly lower mortality from breast cancer than the two unscreened populations. Despite higher mortality due to co-morbidity in the older age group, the death rate due to breast cancer was still significantly higher for older unscreened women than in both screened age groups. In a similar survey of the first age-decade screened patients
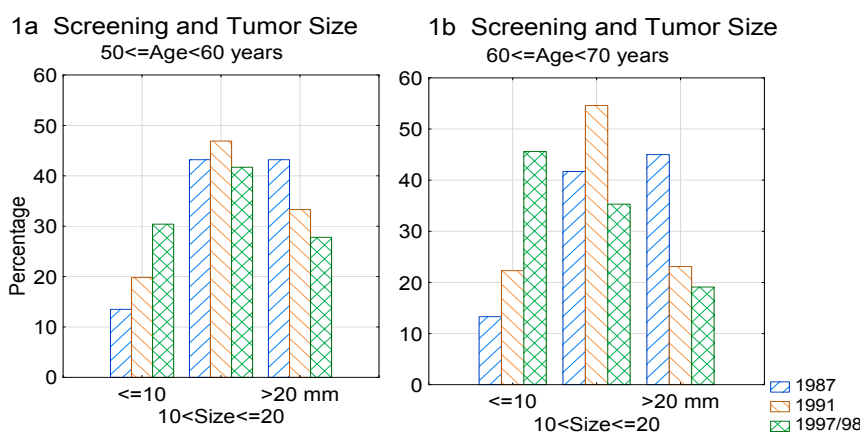

Figure 1: A) Relative distribution of tumor size over three increasing intervals for two mammography screened groups and controls of women aged 50-60 years are shown. Bars represent the percentage for size intervals. For tumors $\leq 10 \mathrm{~mm}$, the 1997/98 sample increased significantly from the control sample of $1987(P<0.01)$ and in the large group $(\geq 20 \mathrm{~mm})$ the tumor size has decreased significantly $(p<0.01)$. B) In the small size group $(\leq 10 \mathrm{~mm})$ the difference has increased further between the screened sample from 1997/98 and the control $(P<0.001)$ and in the large group $(>20 \mathrm{~mm})$ the comparison is also stronger $(P<0.001)$ In the middle size interval the 1991 sample differ significantly both to the control sample $(p<0.05)$ and the $1997 / 98$ sample $(p<0.01)$. In the 1991 sample the large group $(>20 \mathrm{~mm}$ ) decreased significantly related to the contro $(p<0.001)$.
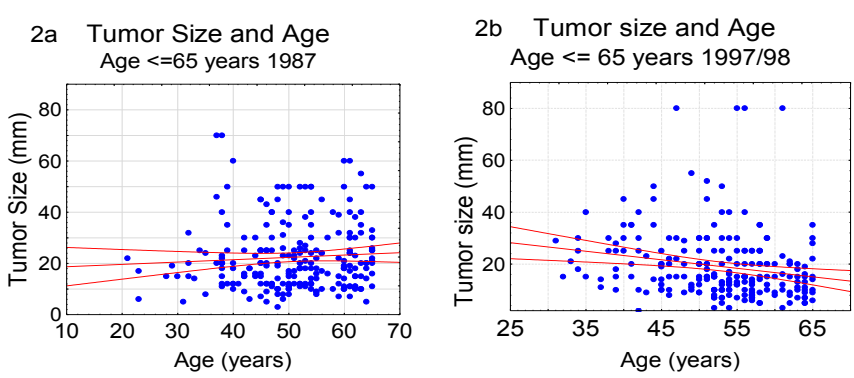

Figure 2: A) A two-dimensional scatter-plot is shown for the control 1987 sample analysis of age against tumors size. A widely dispersed plot is found and linear regression revealed a straight line $(r=0.07 p=0.32 n=231)$. B) In the 1997/98 sample, a two-dimensional scatter-plot based on the 1997/98 sample included unscreened subjects younger than 50 years, to demonstrate the screening effect. Analyzing age against tumor size showed a significant negative slope along with increasing age $(r=-0.20 p<=0.005 n=223)$. The plot reveals the delayed effect in reduction of tumors size from the start of screening at the age of 50 years up to 65 years.

\begin{tabular}{|c|c|c|c|c|c|}
\hline $1997 / 98$ & $\begin{array}{c}<50 \text { years } \\
n=67\end{array}$ & $P$ & $\begin{array}{c}60-69 \text { years } \\
n=70\end{array}$ & $P$ & $\begin{array}{c}\geq 70 \text { years } \\
n=89\end{array}$ \\
\hline $\begin{array}{l}\mathrm{PI} \% \\
\text { (n) }\end{array}$ & $\begin{array}{c}19.78 \pm 16.84 \\
(63)\end{array}$ & $=0.01$ & $\begin{array}{c}13.04 \pm 12.83 \\
(70)\end{array}$ & ns & $\begin{array}{c}13.38 \pm 12.30 \\
(80)\end{array}$ \\
\hline $\begin{array}{l}\text { SSI \% } \\
\text { (n) }\end{array}$ & $\begin{array}{c}23.37 \pm 17.80 \\
(57)\end{array}$ & $<0.05$ & $\begin{array}{c}17.36 \pm 10.91 \\
(67)\end{array}$ & ns & $\begin{array}{c}14.68 \pm 11.86 \\
(80)\end{array}$ \\
\hline $\begin{array}{l}\mathrm{ALNM}^{+} \\
\text {fraction \% } \\
\text { (n) }\end{array}$ & $\begin{array}{c}0.19 \pm 0.28 \\
(56)\end{array}$ & $<0.05$ & $\begin{array}{c}0.11 \pm 0.18 \\
(73)\end{array}$ & ns & $\begin{array}{c}0.13 \pm 0.28 \\
(48)\end{array}$ \\
\hline $\begin{array}{l}\text { Cyclin-A \% } \\
\text { (n) }\end{array}$ & $\begin{array}{c}16.60 \pm 15.26 \\
(67)\end{array}$ & $<0.05$ & $\begin{array}{c}11.64 \pm 13.34 \\
(70)\end{array}$ & ns & $\begin{array}{c}9.26 \pm 9.46 \\
(84)\end{array}$ \\
\hline $\begin{array}{l}\mathrm{Ki} 67 \% \\
\text { (n) }\end{array}$ & $\begin{array}{c}32.53 \pm 24.30 \\
(66)\end{array}$ & ns & $\begin{array}{c}25.81 \pm 25.31 \\
(70)\end{array}$ & ns & $\begin{array}{c}22.4 \pm 22.7 \\
(84)\end{array}$ \\
\hline $\begin{array}{l}\text { Stage I \% } \\
\text { (n) }\end{array}$ & $\begin{array}{l}21.9 \\
(64)\end{array}$ & $<0.05$ & $\begin{array}{l}38.4 \\
(68)\end{array}$ & ns & $\begin{array}{l}22,4 \\
(84)\end{array}$ \\
\hline $\begin{array}{c}\text { Stage IIB } \\
\%(n)\end{array}$ & $\begin{array}{l}17.2 \\
(64)\end{array}$ & $<0.01$ & $\begin{array}{l}2.9 \\
(70)\end{array}$ & ns & $\begin{array}{c}9,4 \\
(84)\end{array}$ \\
\hline
\end{tabular}

Seven parameters - proliferation index (PI), Stemline Scatter Index (SSI); axillary lymph node metastasis (ALNM); cyclin-A, Ki67 and tumor stage I and IIB - were analyzed.. All parameters but Ki67 significantly differed in the younger group. No significant difference appeared against the older patient group. Total number of patients per age interval is shown in the top line of the table. Because the parameters can have some missing values the $n$-values are also shown within the table. For cell cycle parameters the $P$-value is estimated using the mean values \pm $\mathrm{SD}$. For stage I and stage IIB tumors the percentage in relation to the total patient number $(n=64)$ is shown in the table. The P-value is estimated by comparing the percentages related to the $n$-values.

Table 3: Screened woman aged 60-69 years compared with the two unscreened patient groups younger than 50 years and older than 69 years.

aged 50-59 years the mortality from breast cancer was significantly reduced only in the $1997 / 98$ sample versus the younger unscreened group $<50$ years of age $(P<0.05)$ and did differ significantly from the $\geq 70$ year-old unscreened group $(P<0.01)$. The 1991 sample revealed no decrease in death rate from breast cancer compared with the two unscreened age groups for screened patients aged 50-59 years (data not shown). Due to the effect of co-morbidity the two study groups aged 60-69 years differed significantly only to the older group aged $\geq$ 70 years comparing patients alive after 10 years of follow up (Table 4).

\section{Post-screening effect}

First decade after screening: In the first post-screening period, women aged 70-79 years are included. The percentages of tumor $>20$ mm from 1991 and 1997/98 were less than in the control group from 1987 as seen in Figure 3a. This difference compared with controls is 
Citation: Sennerstam RB (2013) During Introduction of Mammography Screening Analysis of Three Tumor Size Intervals in Screened and PostScreened Periods Clarified the Short and Long Term Efficacy of Screening. J Cancer Sci Ther S7: 006. doi:10.4172/1948-5956.S7-006

Page 5 of 7

\begin{tabular}{|c|c|c|c|c|c|}
\hline Age (years) & $<50$ & $P$ & $60-69$ & $P$ & $\geq 70$ \\
\hline 1991 & $\begin{array}{c}96 \\
(100 \%)\end{array}$ & & $142(100 \%)$ & & $169(100 \%)$ \\
\hline Death from BC & $\begin{array}{c}34 \\
(35.4 \%)\end{array}$ & $<0.001$ & $22(15.5 \%)$ & $<0.01$ & $43(25.4 \%)$ \\
\hline Death from other causes & $\begin{array}{c}2 \\
(2.1 \%)\end{array}$ & $<0.01$ & $16(11.3 \%)$ & $<0.001$ & $55(32.5 \%)$ \\
\hline Alive & $\begin{array}{c}60 \\
(62.5 \%)\end{array}$ & ns & $104(73.2 \%)$ & $<0.001$ & $71(42.1 \%)$ \\
\hline $1997 / 98$ & $\begin{array}{c}64 \\
(100 \%)\end{array}$ & & $115(100 \%)$ & & $88(100 \%)$ \\
\hline Dead in BC & $\begin{array}{c}16 \\
(25 \%)\end{array}$ & $<0.001$ & $\begin{array}{c}5 \\
(7.4 \%)\end{array}$ & $<0.01$ & $16(21.6 \%)$ \\
\hline Dead other causes & $\begin{array}{c}2 \\
(3.1 \%)\end{array}$ & $<0.01$ & $\begin{array}{c}9 \\
(13.2 \%)\end{array}$ & $<0.001$ & $34(38.6 \%)$ \\
\hline Alive & $\begin{array}{c}46 \\
(71.9 \%)\end{array}$ & ns & $54(79.4 \%)$ & $<0.001$ & $38(30.8 \%)$ \\
\hline
\end{tabular}

Screened women aged 60-69 years were analyzed for both 1991 and 1997/98 samples for death rates from breast cancer and from other causes. Results were compared with two unscreened age groups, i.e. those younger than 50 years of age and older than 70 years. The 1991 and $1997 / 98$ samples showed significant reductions in breast cancer death rates compared with both the younger $(P<0.001)$ and the older $(P<0.01)$ unscreened patient groups, notwithstanding the co-morbidities of the older patients. The percentage of patients still alive with co-morbidities was, as expected, significantly less in the older patients.

Table 4: Breast cancer death rate and death due to co-morbidity for screened women aged 60-69 years.

close to significance $(P<0.07)$ indicating a lingering post-screening effect. The percentage tumors $10-20 \mathrm{~mm}$ for 1991 continues to be significantly larger than the control (compare Fig $1 b$ ). For tumors $\leq$ $10 \mathrm{~mm}$, another post-screening effect was seen. The high percentage of small tumors (45.6\%) in the 1997/98 sample for screened patients aged $60-69$ years fell to $21,8 \%$ in the first post-screened age group $(P<0.01$; Figure 3a; see Discussion). Only the small tumors $\leq 10 \mathrm{~mm}$ approached control levels immediately after the end of screening; a post-screening effect was seen for the other two size intervals. In the control sample a slight reduction in tumor size $10-20 \mathrm{~mm}$ was seen but compared to the controls aged 50-69 (Figure 1b) no significant difference was found. The percentages patients aged $\geq 70$ years in the three samples were 1987(27\%), 1991 (33.2\%) and 1997/98 (25\%). The 1991 sample increased significantly versus the controls from $1987(\mathrm{P}<0.05)$ and the sample 1997/98 $(\mathrm{P}<0.01)$ indicating a transient increase of post-screened patients in a shorter follow-up in 1991 before the screened patient group aged 50-69 years increases in percentage of whole samples after a longer follow-up: 1987 (48.5\%), 1991 (47.9\%) and $1997 / 98(54.8 \%)(\mathrm{p}<0.05)$
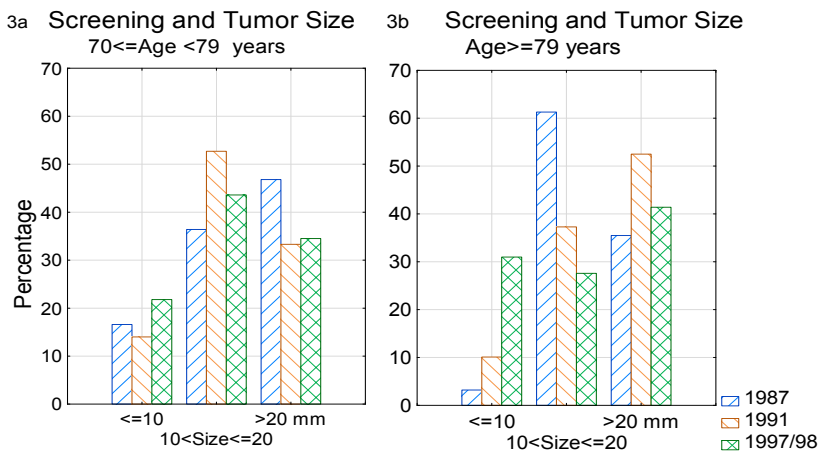

Figure 3: A) Bars represent percentages for each sample in three tumor size interval. For tumors $10-20 \mathrm{~mm}$, the 1991 sample differed significantly from controls $(P<0,05)$. For tumors $\leq 10 \mathrm{~mm}$ the sample from $1997 / 98$ was reduced to the control level. B) For small tumors $\quad(\leq 10 \mathrm{~mm})$ the 1997/98 sample bar increased significantly $(P<0.01)$ related to the controls. In tumors $10-20 \mathrm{~mm}$, the control sample dominates and differs significantly from the1991 $(P<0.05)$ and $1997 / 98(P<0.01)$ samples.

\section{The oldest age group}

In the age group $\geq 79$ years, representing about $10 \%$ in each sample, the control tumors from 1987 for the first time shows a significant change in tumor-size distribution reflected in the bars in Figure 3b. The percentage of control-group tumors $\geq 20 \mathrm{~mm}$ was reduced to $35.5 \%$ as compared with control-group tumors $10-20 \mathrm{~mm}$ $(61.3 \% ; P<0.02)$. These two tumor size intervals in the control sample appeared on an equal level $\sim 40 \%$, for women aged $50-79$ years (Figures $1 \mathrm{a}, 1 \mathrm{~b}$ and $3 \mathrm{~b}$ ). The high percentage control tumors in the $10-20 \mathrm{~mm}$ interval differed significantly from the1997/98 samples (27.6\%), and the corresponding tumor size interval from 1991 (37.3\%; Figure 3b). There is no significant difference found when all three sample in the size interval $\geq 20 \mathrm{~mm}$ are compared (Figure $3 \mathrm{~b}$ ) despite the tending increase in both study samples, probably due to the smaller samples in this age group. Interestingly, tumors $\leq 10 \mathrm{~mm}$ were significantly increased among patients in the 1997/98 sample compared to the controls (see Discussion).

\section{Discussion}

Data from the initial period of mammography screening indicate a lag time before significant positive effect emerged, as reflected in reduced tumor size at diagnosis, decreased genomic instability, lower proliferation activity, diminished involvement of lymph node metastasis reduced death rate due to breast cancer. These benefits showed a clear change first for women aged 60-69 years, when they had attended optimal screening test in the biennial schedule used (Tables 2 and 3). Women aged 50-61 years in 1989 at the start of screening had an optimal chance to be involved in five screening tests, when included in the 1997/98 sample collection at 58-69 years old. In 1991, women aged 55 to 67 years might have the best chance of getting the second mammographic screening that year.

Screening for breast tumors focuses on tumor size. The results show clearly that the other investigated parameters are strongly linked to tumor size. Parameters that reflect growth potentials with cells in S- and G2-phase (PI) and cyclin-A, followed the same pattern to decline significantly for screened patients aged 60-69 years, compared with the unscreened women with breast cancer aged $<50$ years (Table 3 ). For ki67, which indicates cell growth in any cell cycle phase, no 
differences were found. This implies that tumor cells are, as expected, growing in both screened and unscreened patient groups; however, the difference in PI and cyclin-A levels indicate decreased S-phase entry in the smaller diagnosed tumors, which were detected in higher frequency after lengthy screening. For tumor stage I and IIB a swift to significantly increased stage I and decreased stage IIB was found in the screened group aged 60-69 years (Table 3).

Death rate due to breast cancer was significantly diminished in a 10 years of follow up for patients aged 60-69 years compared to unscreened patients aged $<50$ years in both 1991 and $1997 / 98$ samples, as was true versus patients aged $\geq 70$ years (Table 4 ). Notably, a significant higher death rate due to breast cancer appeared in the older patient group despite the higher death rate from other causes [21].

The change in tumor-size distribution in the three size intervals analyzed reflects the impact of mammography screening over time on tumor size (Figures 1 and 3). During the initial introduction period, the frequency of large tumors $>20 \mathrm{~mm}$ was slightly reduced for women 50 59 years in the 1991 sample: However, for women aged 60-69 years, the sample showed a statistically significant reduced percentage of these larger tumors, but still no increase of small tumors $\leq 10 \mathrm{~mm}$ (Figure 1ab). In the $1997 / 98$ sample, included longer screening time at the sample collection, a significant increase in the detection of tumors $\leq 10 \mathrm{~mm}$ was found for women aged 50-59 years as well as a significant reduced frequency of large tumors $>20 \mathrm{~mm}$ (Figure 1a). This trend was more pronounced in women aged 60-69 years (Figure 1b), who attended more screening tests.

Increased incidence of reported breast cancer due to mammography screening and an expected reduced incidence among elderly nonscreened women has been an controversial objective $[1,2,12,13,15,17]$. The idea of detecting tumors by screening that might have regressed spontaneously implies that screening in its early phase harvests many small tumors [15]. However, the present report indicates the contrary: i.e. that screening starts to reduce tumors $\geq 20 \mathrm{~mm}$ the first years (Figures $1 \mathrm{~b}$ and Figure $2 \mathrm{~b}$ ) and increase of tumors $\leq 10 \mathrm{~mm}$ appears in a later phase of screening (Figure 1b). When other variables (such as tumor size, lymph node status and adjustment for lead-time bias) are included, overdiagnosis holds up as an explanation for differences in survival in very few cases [17]. The need for a longer follow-up period has been suggested for estimating overdiagnosis [12]. The significant transient increase in patients aged 60-69 years soon after introduction of screening in the 1991 sample and the significant increase in the screened group aged 50-69 years after some more years of screening in the 1997/98 sample is an observation that needs further analysis in larger samples.

The increased transient Stage II+ found during the first years after introduction of screening [14] was interpreted as a candidate for spontaneously tumor regression. That result might correspond to the transient increase in Stage IIB T1c found in the 1991 sample, during the introduction period of screening, due to the redistribution within the three tumor size intervals (Figure 1b).

A post-screening effect was found in this report. Both investigated populations from 1991 and 1997/98 had a decreased percentage of tumors $>20 \mathrm{~mm}$ for women aged 70-79 years as a lingering effect after screening. Between screened women aged 60-69 years and the first post-screened group aged 70-79 years the tumors $\leq 10 \mathrm{~mm}$ in the $1997 / 98$ sample deceased significantly from $45.5 \%$ to $21.8 \%$-i.e. back to the control levels (Figure 3a). This decline in tumors $\leq 10 \mathrm{~mm}$ after the screening period might be one part of an explanation for not observing an increased incidence of breast cancer cases after the end of the screening period.

The bars of the control sample from 1987 in Figures 1a and 1b did not change in size distribution at all between the three tumorsize intervals for women aged 50-69 years. A slight but not significant reduction in intermediate tumor size for controls aged 70-79 years was seen which indicates that breast self-examination (BSE) and clinical breast examination (CBE) did not change the distribution of control tumor size sub-groups in the age interval 50-79 years. However, it was observed in the oldest age group $\geq 79$ years.

In the oldest population, control size distribution changed (Figure $3 \mathrm{~b})$. The percentage of control tumors $>20 \mathrm{~mm}$ decreased for women aged $\geq 79$ years, while control tumors $10-20 \mathrm{~mm}$ increased significantly (Figure 3b). It is similar to the 1991 sample and the tumor-size distribution for screened patients aged 60-69 years (Fig.1b) reflecting an early effect of screening before the increase in tumor size $\leq 10 \mathrm{~mm}$ appears. BSE and CBE may affect control tumor-size distribution at diagnosis at this high age, thus reducing the percentage of tumors $>$ $20 \mathrm{~mm}$. The increase in the percentage of tumors $\leq 10 \mathrm{~mm}$ at this high age, found in the 1997/98 sample, might be a late compensating postscreening effect, when small tumors re-appear after being diagnosed in high frequency during screening. At this high age, they have reached a now-palpable size and are more easily detected in aging breast tissues with decreased fat involvement (Figure 3b).

\section{Conclusions}

These results analyze screening effect on three tumor-size intervals 2 and 8 years after introduction of mammographic screening. They show that during the first period mainly large tumor $20 \mathrm{~mm}$ are diagnosed and as screening proceeds, discovery of smaller tumors $\leq 10$ $\mathrm{mm}$ increases. These results imply that diagnosis of small but clinically significant tumors through screening requires long-term followup, and might not be most effective the first years after screening is introduced. The method used in this report reveals a post-screening effect of drastic reducing in the frequency of small tumors $\leq 10 \mathrm{~mm}$ and still diagnosing tumors $>20 \mathrm{~mm}$ at rates smaller than those of controls, as a lingering post-screening effect in the first age decade after screening. Further research might address whether the delayed effect of screening in reducing tumor size is related to the fact that more and earlier X-rays facilitate detection of change by allowing comparison of previous images. Improvement of screening over time implies that longer screening periods give stronger screening benefits. Increasing the screening period in Sweden from 40 years to 74 years has reduced the mean tumor size to $17 \mathrm{~mm}$. The question of overdiagnosis is an important issue that needs further studies to reach consensus.

\section{Acknowledgements}

Fruitful discussions have been held with prof Edward Azavedo, Radiology Department, Section of Mammography, Karolinska Hospital and Karolinska Institutet, Stockholm, Sweden. Edanz Group Ltd did language editing. Support given from Karolinska Institutet Funds.

\section{Conflict of Interest}

This report does not include the use of any commercial products with connections to the author. The staining techniques used have been established since several decades. There is no way to get economical benefits from a publication of this article

\section{References}

1. Jørgensen KJ, Gøtzsche PC (2009) Overdiagnosis in publicly organised 
Citation: Sennerstam RB (2013) During Introduction of Mammography Screening Analysis of Three Tumor Size Intervals in Screened and PostScreened Periods Clarified the Short and Long Term Efficacy of Screening. J Cancer Sci Ther S7: 006. doi:10.4172/1948-5956.S7-006

Page 7 of 7

mammography screening programmes: systematic review of incidence trends. BMJ 339: b2587

2. Puliti D, Duffy SW, Miccinesi G, de Koning H, Lynge E, et al. (2012) Overdiagnosis in mammographic screening for breast cancer in Europe: a literature review. J Med Screen 19 Suppl 1: 42-56.

3. Jonsson $H$, Johansson $R$, Lenner $P$ (2005) Increased incidence of invasive breast cancer after the introduction of service screening with mammography in Sweden. Int J Cancer 117: 842-847.

4. Zahl PH, Strand BH, Maehlen J (2004) Incidence of breast cancer in Norway and Sweden during introduction of nationwide screening: prospective cohort study. BMJ 328: 921-924.

5. Tabár L, Fagerberg CJ, Gad A, Baldetorp L, Holmberg LH, et al. (1985) Reduction in mortality from breast cancer after mass screening with mammography. Randomised trial from the Breast Cancer Screening Working Group of the Swedish National Board of Health and Welfare. Lancet 1: 829832.

6. Tabar L, Fagerberg G, Chen HH, Duffy SW, Smart CR, et al. (1995) Efficacy of breast cancer screening by age. New results from the Swedish Two-County Trial. Cancer 75: 2507-2517.

7. Autier P, Koechlin A, Smans M, Vatten L, Boniol M (2012) Mammography screening and breast cancer mortality in Sweden. J Natl Cancer Inst 104: 10801093.

8. Wishart GC, Greenberg DC, Britton PD, Chou P, Brown CH, et al. (2008) Screen-detected vs symptomatic breast cancer: is improved survival due to stage migration alone? $\mathrm{Br} \mathrm{J}$ Cancer 98: 1741-1744.

9. Dawson SJ, Duffy SW, Blows FM, Driver KE, Provenzano E, et al. (2009) Molecular characteristics of screen-detected vs symptomatic breast cancers and their impact on survival. Br J Cancer 101: 1338-1344.

10. Lawrence G, O'Sullivan E, Kearins O, Tappenden N, Martin K, et al. (2009) Screening histories of invasive breast cancers diagnosed 1989-2006 in the West Midlands, UK: variation with time and impact on 10-year survival. J Med Screen 16: 186-192.

11. Coburn NG, Cady B, Fulton JP, Law C, Chung MA (2012) Improving size lymph node metastatic rate, breast conservation, and mortality of invasive breast cancer in Rhode Island women, a well-screened population. Breast Cancer Res Treat 135: 831-837.

12. Falk RS, Hofvind S, Skaane P, Haldorsen T (2013) Overdiagnosis among women attending a population-based mammography screening program. Int J Cancer 133: 705-712.

13. Paci E, Duffy S (2005) Overdiagnosis and overtreatment of breast cancer: overdiagnosis and overtreatment in service screening. Breast Cancer Res 7 266-270.

14. Frisell J, Eklund G, Hellström L, Glas U, Somell A (1989) The Stockholm breast cancer screening trial--5-year results and stage at discovery. Breast Cancer Res Treat 13: 79-87.

15. Zahl PH, Gøtzsche PC, Mæhlen J (2011) Natural history of breast cancers detected in the Swedish mammography screening programme: a cohort study. Lancet Oncol 12: 1118-1124.

16. Román R, Sala M, Salas D, Ascunce N, Zubizarreta R, et al. (2012) Effect of protocol-related variables and women's characteristics on the cumulative falsepositive risk in breast cancer screening. Ann Oncol 23: 104-111.

17. Allgood PC, Duffy SW, Kearins O, O'Sullivan E, Tappenden N, et al. (2011) Explaining the difference in prognosis between screen-detected and symptomatic breast cancers. Br J Cancer 104: 1680-1685.

18. Crispo A, Barba M, D’Aiuto G, De Laurentiis M, Grimaldi M, et al. (2013) Molecular profiles of screen detected vs. symptomatic breast cancer and their impact on survival: results from a clinical series. BMC Cancer 13: 15.

19. Shapiro S, Coleman EA, Broeders M, Codd M, de Koning H, et al. (1998) Breast cancer screening programmes in 22 countries: current policies, administration and guidelines. International Breast Cancer Screening Network (IBSN) and the European Network of Pilot Projects for Breast Cancer Screening. Int J Epidemiol 27: 735-742.

20. Kronenwett U, Huwendiek S, Ostring C, Portwood N, Roblick UJ, et al. (2004 Improved grading of breast adenocarcinomas based on genomic instability. Cancer Res 64: 904-909.

21. Sennerstam RB, Wiksell H, Schässburger KU, Auer GU (2012) Breast cance and clinical outcome among women over 60 years of age: a plead for more screening and alternative treatments. Anal Quant Cytol Histol 34: 189-194.
This article was originally published in a special issue, Cancer Screening \& Early Detection handled by Editor(s). Dr. Arutselvan Natarajan, Stanford University, United States 